This item was submitted to Loughborough's Research Repository by the author.

Items in Figshare are protected by copyright, with all rights reserved, unless otherwise indicated.

\title{
A finite-volume-based two-dimensional wall-flow diesel particulate filter regeneration model
}

PLEASE CITE THE PUBLISHED VERSION

http://dx.doi.org/10.1243/09544070JAUTO680

PUBLISHER

Sage (@ IMechE)

VERSION

AM (Accepted Manuscript)

\section{PUBLISHER STATEMENT}

This work is made available according to the conditions of the Creative Commons Attribution-NonCommercialNoDerivatives 4.0 International (CC BY-NC-ND 4.0) licence. Full details of this licence are available at: https://creativecommons.org/licenses/by-nc-nd/4.0/

\section{LICENCE}

CC BY-NC-ND 4.0

\section{REPOSITORY RECORD}

Law, Ming-Chiat, Andrew Clarke, Colin P. Garner, and Andrew M. Williams. 2014. "A Finite-volume-based Two-dimensional Wall-flow Diesel Particulate Filter Regeneration Model”. figshare. https://hdl.handle.net/2134/15684. 


\title{
A finite-volume based two-dimensional wall-flow
}

\section{diesel particulate filter regeneration model}

\author{
M. C. Law, A. Clarke, C. P. Garner, A. M. Williams*
}

Wolfson School of Mechanical and Manufacturing Engineering, Loughborough University, Loughborough, LE11 3TU, UK

"Corresponding author: Wolfson School of Mechanical and Manufacturing Engineering, Loughborough, Leicestershire, LE11 3TU, UK. Email: A.M.Williams@Lboro.ac.uk

\begin{abstract}
Many existing diesel particulate filter (DPF) models do not sufficiently describe the actual physio-chemical processes that occur during the regeneration process. This is due to the various assumptions made in the models. To overcome this shortcoming, a detailed two-dimensional (2-D) DPF regeneration model with a multistep chemical reaction scheme is presented. The model solves the variable density, multicomponent conservation equations by PISO (Pressure Implicit with Splitting of Operators) scheme for inlet and outlet channels as well as the porous soot layer and filter wall. It includes a non-thermal equilibrium (NTE) model for the energy equation for porous media. In addition, for the first time, experiments on DPF were conducted to determine interstitial heat transfer coefficient inside the DPF porous wall. The results compare well with an in-house 1-D model and subsequently this was used in the new 2-D model. By using this detailed 2-D model, some interesting observations of the DPF regeneration process were revealed. These included flow reversals and asymmetry in the filters channels.
\end{abstract}

Keywords: diesel, engine, particulate, filter, trap, regeneration, soot, chemical reactions, non-thermal equilibrium, PISO, model.

\section{INTRODUCTION}

Modern diesel engine-driven vehicles are well known for their durability and good fuel economy (low carbon footprint) and this has resulted in increasing market share, especially in Europe. However, diesel engines suffer from high emissions of particulate 
matter $(\mathrm{PM})$ and oxides of nitrogen $\left(\mathrm{NO}_{\mathrm{x}}\right)$ which are associated with environmental pollution and potential health hazards $[1,2]$. Consequently, increasingly stringent emission legislation, such as EURO V and US Tier 3, have been introduced to limit harmful emissions. It has been predicted that diesel particulate filters (DPFs), which are able to retain the diesel PM inside the filter will become a necessity in order to reduce PM emissions to the regulated levels. Advances in DPF technology have enabled them to be used widely since 2001 [3]. Diesel PM that is retained inside a DPF needs to be oxidised by either internal or external measures [3] to avoid excessive exhaust gas back pressure which not only adversely affects engine power but also fuel economy. The PM oxidation process inside a DPF is known as regeneration. The DPF regeneration process has to be well-controlled so that the resulting heat release will not damage the DPF material. Experimental optimisation of DPF systems is a costly and time-consuming task, hence a DPF regeneration model is valuable not only to better understand the physics of the regeneration process but also as a design tool to devise better regeneration strategies.

Many DPF regeneration models have been developed in the past two decades. These range from zero- to three-dimensional (3-D) models [4-11]. Recent years have seen the development of 3-D DPF models at full filter scale $[\mathbf{8}, \mathbf{9}, \mathbf{1 2}]$. In these approaches, DPF channels were modelled by a set of one-dimensional (1-D) equations, as developed by Bissett [13]. The solutions were then used for the calculation of the three-dimensional energy equation for the whole filter. Hou and Angelo [14] presented a 3-D regeneration model at the channel / wall scale. The authors neglected changes in gas density during the regeneration process and assumed thermal equilibrium (TE) in the PM layer and the filter wall.

Conversely, there are limited physio-chemical models for the DPF regeneration processes at the channel scale. Therefore, this paper presents a new two-dimensional (2D) DPF model to help provide more details. The present model calculates a transient, multi-component, variable-density flow and assumes a non-thermal equilibrium (NTE) combustion model for porous media (PM layer and filter wall). It also incorporates a multi-step chemical reaction scheme for thermal PM oxidation as reported previously [11]. In addition, the paper also presents a novel heat transfer experiment that was conducted to estimate, for the first time, the interstitial heat transfer coefficient inside the DPF filter wall. This important parameter was then used in the new 2-D model. 
The next section of the paper will discuss the new 2-D model in detail. Subsequent sections will present the experimental work to determine the interstitial heat transfer coefficient of a clean wall-flow DPF. The results of the new 2-D model are then compared with data reported in the literature and lastly, the main findings of the paper are summarised.

\section{DPF REGENERATION MODEL}

\subsection{Description of Model}

Figure 1 illustrates the domain of interest, which consists of half of the inlet and outlet channels as well as the PM layer and filter wall. The three distinct media that constitute the domain of interest in the current model are the free stream exhaust gas flow, consisting of inlet and outlet channels, the porous media of the PM layer and the filter wall. These three media can be modelled as two media with varying properties - the porous structure and the open structure. The exhaust gas flows into the filter through an inlet channel and is forced through the porous PM layer and wall due to the end plug at the downstream end of the inlet channel. The inlet flow rate of PM is $\sim 1 \%$ the PM oxidation rate and, therefore, is not significant enough to affect the PM oxidation event. It finally exits via the outlet channel. Since each inlet channel is surrounded by four identical porous walls, the gas is assumed to be drawn into the adjacent outlet channels evenly. This promotes a symmetrical flow field and therefore only half of the channel width needs to be considered in the computational domain. The two dimensional geometry represents an infinite length in the $\mathrm{z}$ direction which, although it does not model two out of the four channels, it does give another level of understanding to the thermal, chemical and flow characteristics of diesel particulate filter regeneration beyond previous one dimensional models [??].

$<$ Figure 1 $>$ 


\subsection{General Flow Field Solution (2-D)}

\subsubsection{Free Stream}

The continuity equation for free stream variable density flow (due to changes in gas pressure and temperature) is given by

$$
\frac{\partial\left(\rho_{G}\right)}{\partial t}+\frac{\partial\left(\rho_{G} u\right)}{\partial x}+\frac{\partial\left(\rho_{G} v\right)}{\partial y}=0
$$

The gas phase momentum equations are given as

$$
\begin{aligned}
& \frac{\partial\left(\rho_{G} u\right)}{\partial t}+\frac{\partial\left(\rho_{G} u \cdot u\right)}{\partial x}+\frac{\partial\left(\rho_{G} v \cdot u\right)}{\partial y}=-\frac{\partial p}{\partial x}+\frac{\partial}{\partial x}\left\{\mu_{G} \frac{\partial u}{\partial x}\right\}+\frac{\partial}{\partial y}\left\{\mu_{G} \frac{\partial u}{\partial y}\right\} \\
& \frac{\partial\left(\rho_{G} v\right)}{\partial t}+\frac{\partial\left(\rho_{G} u \cdot v\right)}{\partial x}+\frac{\partial\left(\rho_{G} v \cdot v\right)}{\partial y}=-\frac{\partial p}{\partial y}+\frac{\partial}{\partial x}\left\{\mu_{G} \frac{\partial v}{\partial x}\right\}+\frac{\partial}{\partial y}\left\{\mu_{G} \frac{\partial v}{\partial y}\right\}
\end{aligned}
$$

where $\mu_{G}$ is the exhaust gas dynamic viscosity.

\subsubsection{Porous Media - PM Layer and Filter Wall}

The continuity equation for a porous medium can be written as

$$
\frac{\partial\left(\phi \rho_{G}\right)}{\partial t}+\frac{\partial\left(\rho_{G} u_{D}\right)}{\partial x}+\frac{\partial\left(\rho_{G} v_{D}\right)}{\partial y}=\sum M W_{\eta} \cdot \sum\left[\phi \cdot \dot{R}_{G, \alpha}^{\prime \prime \prime}+\dot{R}_{S, \alpha}^{\prime \prime \prime}\right]
$$

The Darcian (average) velocity, $\mathbf{u}_{D}\left(u_{D}, v_{D}\right)$ in the porous media can be related to the pore velocity via $\mathbf{u}_{D}\left(u_{D}, v_{D}\right)=\phi \cdot \mathbf{u}_{\text {porr }}\left(u_{\text {porr }}, v_{\text {porr }}\right)$. There is a mass addition term, $\sum\left[\phi \cdot \dot{R}_{G, \alpha}^{\prime \prime \prime}+\dot{R}_{S, \alpha}^{\prime \prime \prime}\right]$ in equation (4) owing to the gas and solid phase reactions in the PM cake layer. However, there is no mass addition in the porous filter wall. 
To account for the exhaust gas flow in the porous wall and PM layer, the generalised Brinkman-Forchheimer-extended Darcy model [15] is used and these are given, in x- and y-direction, as

$\mathrm{x}$-momentum

$$
\begin{aligned}
& \frac{\partial\left(\phi \rho_{G} u_{D}\right)}{\partial t}+\frac{\partial\left(\rho_{G} u_{D} u_{D}\right)}{\partial x}+\frac{\partial\left(\rho_{G} u_{D} v_{D}\right)}{\partial y} \\
& =-\phi^{2} \frac{\partial p}{\partial x}+\frac{\partial}{\partial x}\left\{\mu_{e f f} \frac{\partial u_{D}}{\partial x}\right\}+\frac{\partial}{\partial y}\left\{\mu_{e f f} \frac{\partial u_{D}}{\partial y}\right\}-\phi^{2}\left(\frac{\mu_{G}}{K}+\frac{C_{E}}{K^{1 / 2}} \rho_{G}\left\|\mathbf{U}_{D}\right\|\right) u_{D}
\end{aligned}
$$

y-momentum

$$
\begin{aligned}
& \frac{\partial\left(\phi \rho_{G} v_{D}\right)}{\partial t}+\frac{\partial\left(\rho_{G} u_{D} v_{D}\right)}{\partial x}+\frac{\partial\left(\rho_{G} v_{D} v_{D}\right)}{\partial y} \\
& =-\phi^{2} \frac{\partial p}{\partial y}+\frac{\partial}{\partial x}\left\{\mu_{e f f} \frac{\partial v_{D}}{\partial x}\right\}+\frac{\partial}{\partial y}\left\{\mu_{e f f} \frac{\partial v_{D}}{\partial y}\right\}-\phi^{2}\left(\frac{\mu_{G}}{K}+\frac{C_{E}}{K^{1 / 2}} \rho_{G}\left\|\mathbf{U}_{D}\right\|\right) v_{D}
\end{aligned}
$$

where $\mu_{e f f}=\phi \mu_{G}, K$ is the permeability, $C_{E}$ is the inertial term for the porous media and $\left\|\mathbf{U}_{D}\right\|$ is the magnitude of the resultant Darcian velocity. These terms are given as: $K_{p}=1.8 \times 10^{-14} \mathrm{~m}^{2}$ for PM layer [16] and $K_{f j}=\frac{\phi_{f w}^{3}}{150 \cdot\left(1-\phi_{f w}^{2}\right)} d_{f w}^{2} \mathrm{~m}^{2}$ [17] for filter wall, $C_{E}=\frac{1.75}{\sqrt{150}} \phi^{-1.5}[\mathbf{1 8}]$ (for either the PM layer or the filter wall) and $\left\|\mathbf{U}_{D}\right\|=\sqrt{u^{2}+v^{2}}$.

\subsection{Temperature Field}

There are three main mechanisms of energy transfer in a DPF; conduction, convection and radiation. Prior to the onset of regeneration process, the inlet gas temperature is the main heat source of the whole DPF. To initiate regeneration, the gas temperature is increased to the ignition temperature of diesel PM. At this stage, the energy transfer is mainly from the gas phase to the solid phase. Once the regeneration process occurs, the heat liberated from PM oxidation is so high that the resultant / net heat flow is from the solid phase to the gas phase via convection. At the same time, the heat source is also transported to the whole DPF via the PM layer and the filter wall conduction. Since DPF 
channels are slender, the four surfaces in each channel are assumed to exchange equal amount of radiative heat [19]. Hence, radiative heat loss is neglected in the model.

\subsubsection{Free Stream Energy Equation}

In this section, the energy equation for the free stream in the inlet and the outlet channels is presented. For the porous media, NTE is implemented in the model. The energy equation for the inlet and the outlet channels is given as

$$
\begin{aligned}
& \frac{\partial\left(\rho_{G} c_{p G} T_{G}\right)}{\partial t}+\frac{\partial\left(\rho_{G} c_{p G} u T_{G}\right)}{\partial x}+\frac{\partial\left(\rho_{G} c_{p G} v T_{G}\right)}{\partial y} \\
& =\frac{\partial}{\partial x}\left\{k_{G} \frac{\partial T_{G}}{\partial x}\right\}+\frac{\partial}{\partial y}\left\{k_{G} \frac{\partial T_{G}}{\partial y}\right\}+\sum_{\alpha} \dot{R}_{G, \alpha}^{\prime \prime \prime} \cdot H_{G, \alpha}
\end{aligned}
$$

On the left hand side of equation (7) are transient and convective terms in $\mathrm{x}$ - and $\mathrm{y}$ directions. These are balanced by a fluid conduction term and a heat source term on the right hand side. The term $\sum_{\alpha} \dot{R}_{G, \alpha}^{\prime \prime \prime} \cdot H_{G, \alpha}$ is the sum of the product of gas phase reaction rates, $\alpha$, and the corresponding enthalpies of reaction.

\subsubsection{Non-Thermal Equilibrium (NTE) Energy Equations}

Many of the existing DPF models (such as those developed as in [7], [10] and [14]) assumed TE for the porous media (PM layer and filter wall) temperature calculations. Zheng and Keith [10] argued that since the specific area in the DPF was generally large, the TE assumption was justifiable. Nevertheless, Kaviany [15] warned that the TE assumption in the porous media was violated if there was a heat generation source in either the solid or the gas phase. Hence, the present study investigates the validity of TE in the case of DPF regeneration by developing a non-thermal equilibrium (NTE) model, which consists of gas and solid phase energy equations.

The NTE temperature equations are as follows

Gas phase 


$$
\begin{aligned}
& \frac{\partial\left(\phi \rho_{G} c_{p G} T_{G}\right)}{\partial t}+\frac{\partial\left(\rho_{G} c_{p G} u_{D} T_{G}\right)}{\partial x}+\frac{\partial\left(\rho_{G} c_{p G} v_{D} T_{G}\right)}{\partial y} \\
& =\frac{\partial}{\partial x}\left\{\phi k_{G} \frac{\partial T_{G}}{\partial x}\right\}+\frac{\partial}{\partial y}\left\{\phi k_{G} \frac{\partial T_{G}}{\partial y}\right\}+h_{\text {interstitial }} \cdot a \cdot\left(T_{S}-T_{G}\right)+\sum_{\alpha}\left(\phi \cdot \dot{R}_{G, \alpha}^{\prime \prime \prime} \cdot H_{G, \alpha}\right)
\end{aligned}
$$

Solid phase

$$
\begin{aligned}
& \frac{\partial\left[\beta_{S} c_{p S} T_{S}\right]}{\partial t} \\
& =\frac{\partial}{\partial x}\left\{k_{S} \frac{\partial T_{S}}{\partial x}\right\}+\frac{\partial}{\partial y}\left\{k_{S} \frac{\partial T_{S}}{\partial y}\right\}+h_{\text {interstitial }} \cdot a \cdot\left(T_{G}-T_{S}\right)+\sum_{\alpha}\left(\dot{R}_{S, \alpha}^{\prime \prime \prime} \cdot H_{S, \alpha}\right)
\end{aligned}
$$

The coupling between gas and solid phases at temperature $T_{G}$ and $T_{S}$ respectively is via the volumetric interstitial heat transfer term, $b_{\text {interstitial }} \cdot a$. This value was determined experimentally for a ceramic DPF and is described in Section 4.

\subsection{Gas Species Field}

Since the PM reacts with gas species during the regeneration process, the concentrations of gas species vary throughout the computational domain. In addition, the reaction rate is dependent on the oxidant concentrations, e.g. oxygen, therefore, it is crucial that the model is able to predict accurately the species profile in the DPF. The gas species equation inside a porous medium can be written as

$$
\begin{aligned}
& \frac{\partial\left(\phi \rho_{G} Y_{\eta}\right)}{\partial t}+\frac{\partial\left(\rho_{G} Y_{\eta} u_{D}\right)}{\partial x}+\frac{\partial\left(\rho_{G} Y_{\eta} v_{D}\right)}{\partial y} \\
& =\frac{\partial}{\partial x}\left(\rho_{G} D_{e f f, \eta} \frac{\partial Y_{\eta}}{\partial x}\right)+\frac{\partial}{\partial y}\left(\rho_{G} D_{e f f, \eta} \frac{\partial Y_{\eta}}{\partial y}\right)+M W_{\eta} \cdot \sum_{\alpha} v_{\eta \alpha}\left[\phi \cdot \dot{R}_{G, \alpha}^{\prime \prime \prime}+\dot{R}_{S, \alpha}^{\prime \prime \prime}\right]
\end{aligned}
$$

where $D_{e f f, \eta}$ is the effective diffusion coefficient of a gas species $\eta$ inside a porous medium. Equation (10) consists of transient and convective terms on the left hand side and the diffusive and reaction source terms are on the right hand side. This equation can be modified for a free-stream case by setting $\phi=1, \dot{R}_{S, \alpha}^{\prime \prime \prime}=0$ and $D_{e f f, \eta}=D_{\eta_{m}}$ where $D_{\eta m}$ 
is the diffusion of species $\eta$ in the gas mixture. The term $Y_{\eta}$ is the mass fraction of gas species $\eta$. This equation is applicable to all the individual gas species, i.e. $\eta=1,2,3, \ldots, N-1, N$, where $N$ is the total number of gas species. However, due to the principle of mass conservation the computation is only required for $\mathrm{N}-1$ species.

\subsubsection{Free Stream}

In practical situations, the concentrations of gas species are not in equilibrium throughout the DPF. Due to factors such as concentration, pressure and temperature gradients, the gas molecules diffuse towards one another, even if there is no convective flow. In the present case, six species have been considered as taking part in the multi-step chemical reaction scheme. Therefore, multi-component diffusion needs to be taken into account.

For this study only the diffusion caused by the gas concentration gradient is considered (i.e. ordinary diffusion). Other factors such as temperature and pressure gradients in the free stream are neglected in the present study. The diffusion coefficient of species $\eta$ in the gas mixture, $D_{\eta m}$ can be calculated from

$$
D_{\eta m}=\frac{1-\chi_{\eta}}{\sum_{\gamma \neq \eta}^{N} \frac{\chi_{\gamma}}{D_{\eta \gamma}}}
$$

where $\chi_{\eta}$ is the molar fraction of species $\eta[20]$, which in turn can be calculated from $\chi_{\eta}=Y_{\eta} \cdot\left(\frac{M W_{\text {mix }}}{M W_{\eta}}\right)$ and the molecular weight of gas mixture is $M W_{\text {mix }}=\sum_{\eta} \chi_{\eta} \cdot M W_{\eta}$.

\subsubsection{Porous Medium}

The diffusion coefficient in a porous medium needs to be modified in order to account for the narrow and irregular path inside the porous media. The gas motion inside the porous media is known as Knudsen diffusion. The diffusion coefficient is defined as [21] 


$$
D_{\text {Knud }, \eta}=\frac{d_{p}}{3} \sqrt{\frac{8 \mathrm{R}_{u} T_{G}}{\pi \cdot M W_{\eta}}}
$$

where $d_{p}$ is the mean pore diameter, $R_{u}$ is the universal gas constant and $M W_{\eta}$ is the molecular weight of gas species $\eta$.

The overall effective diffusion coefficient is thus [21]

$$
D_{e f f, \eta}=\frac{\phi}{\tau}\left[\frac{D_{K n u d, \eta} \cdot D_{\eta m}}{D_{K n u d, \eta}+D_{\eta m}}\right]
$$

where $\tau$ is the tortuosity factor which is the ratio of the distance between two points travelled by a molecule in an irregular path in a porous medium to the line of sight distance between these two points [22]. In this case, the value is taken to be 3 [23].

\subsection{PM Oxidation Rate}

The rate of PM oxidation is found from the sum of heterogeneous reactions that occurs and this is represented by

$$
\frac{\partial \beta_{p}}{\partial t}=-M W_{p} \sum_{\alpha} v_{p \alpha} \cdot \dot{R}_{S, \alpha}^{\prime \prime \prime}
$$

where $\beta_{p}$ is the PM bulk density and $v_{p \alpha}$ is the stoichiometric coefficient of reaction $\alpha$.

\subsection{Equation of State}

The model is completed by adding the equation of state to relate the gas density variation with respect to pressure, $p$ and gas temperature, $T_{G}$. This is given by

$$
\rho=\frac{p}{R_{G} T_{G}}
$$

The specific gas constant $R_{G}$ can be found by dividing $R_{u}$ with the mean molecular weight of gas mixture. 


\subsection{Chemical Reaction Scheme}

Since only the thermal PM oxidation is studied in this paper, the global reactions involved are as follows

$$
\begin{aligned}
& \mathrm{C}+\frac{1}{2} \mathrm{O}_{2} \rightarrow \mathrm{CO} \\
& \mathrm{CO}+\frac{1}{2} \mathrm{O}_{2} \Leftrightarrow \mathrm{CO}_{2} \\
& \mathrm{C}+\mathrm{O}_{2} \rightarrow \mathrm{CO}_{2} \\
& \mathrm{CO}+\mathrm{H}_{2} \mathrm{O} \Leftrightarrow \mathrm{CO}_{2}+\mathrm{H}_{2}
\end{aligned}
$$

Heterogeneous reactions, R1 and R3 are one-way forward reactions whilst homogeneous gas phase reactions $\mathrm{R} 2$ and $\mathrm{R} 4$ (water gas shift reaction) are reversible reactions. The

\begin{tabular}{|c|c|c|c|c|}
\hline Reaction & Rate Expression & Pre-exponential constant, $A$ & Activation energy, $E$ & Ref \\
\hline R1 & $\dot{R}_{S, 1}^{\prime \prime \prime}=\frac{k_{1} b}{k_{1}+b}\left[O_{2}\right]$ & $A_{1}=2.64 \times 10^{7} \mathrm{~s}^{-1}$ & $E_{1}=79.791 \mathrm{~kJ} \mathrm{~mol}^{-1}$ & [??] \\
\hline R2 & $\dot{R}_{G, 2}^{\prime \prime \prime}=k_{2 f}\left[\mathrm{CO} \llbracket\left[\mathrm{H}_{2} \mathrm{O}\right]^{0.5}\left[\mathrm{O}_{2}\right]^{0.3}-k_{2 r}\left[\mathrm{CO}_{2}\right]\right.$ & $A_{2}=3.8 \times 10^{6} \mathrm{~s}^{-1}$ & $E_{2}=66.88 \mathrm{~kJ} \mathrm{~mol}^{-1}$ & [??] \\
\hline $\bar{R} 3$ & $\dot{R}_{S, 3}^{\prime \prime \prime}=\frac{k_{3} b}{k_{3}+b}\left[O_{2}\right]$ & $A_{3}=3.68 \times 10^{6} \mathrm{~s}^{-1}$ & $E_{3}=142 \mathrm{~kJ} \mathrm{~mol}^{-1}$ & [??] \\
\hline R4 & $\dot{R}_{G, 4}^{\prime \prime \prime}=\frac{k_{4 f}\left[H_{2} O \|[C O]^{0.5}\right.}{\left(1+379.473\left[H_{2}\right]^{0.5}\right.}-\frac{k_{4 r}\left[\mathrm{CO}_{2}\left[\mathrm{H}_{2}\right]^{0.5}\right.}{1+3.6[\mathrm{CO}]}$ & $\begin{array}{l}A_{4 f}=1.58 \times 10^{11} \mathrm{~m}^{3 / 2} \mathrm{~mol}^{-1 / 2} \mathrm{~s}^{-1} \\
A_{4 r}=3 \times 10^{9} \mathrm{~m}^{3 / 2} \mathrm{~mol}^{-1 / 2} \mathrm{~s}^{-1}\end{array}$ & $\begin{array}{l}E_{4 f}=281.314 \mathrm{~kJ} \mathrm{~mol}^{-1} \\
E_{4 \mathrm{r}}=238.26 \mathrm{~kJ} \mathrm{~mol}^{-1}\end{array}$ & [??] \\
\hline
\end{tabular}
kinetic parameters of these reactions were reported in [11] and are shown here in Table ??. In Equation (??) the gas phase contains only the homogeneous reactions while the solid (porous) phase includes the heterogeneous reaction terms.

\subsection{Boundary Conditions}

The boundary conditions of the computational domain are as follows:

\section{Inflow}


At the inlet, the axial velocity was calculated from the inlet mass flow rate and since the inlet radial velocity was usually not known, it was set to zero, i.e. $u=U_{i n}, v=0$. Other scalar variables such as species mass fraction and gas temperature were taken as typical exhaust gas conditions.

\section{Symmetry line}

The shear stress in the u-momentum equation was set to zero, following [24], whereas for v-momentum, $v_{\text {symmetry }}$ was zero at the line of symmetry. For scalar variables, zero normal gradients were assumed. Hence, the boundary conditions were

$$
\frac{\partial u}{\partial y}=0, \quad v_{\text {symmetry }}=0, \quad \frac{\partial T_{G}}{\partial y}=0 \quad \text { and } \quad \frac{\partial Y_{\eta}}{\partial y}=0
$$

\section{Plugged wall}

No flow boundary conditions were applied to the plug wall surface. The heat transfer from to and from the plug is considered to have a negligible effect on the oxidation event and hence is considered a thermal insulation boundary. Thus

$$
u=0, \quad \frac{\partial v}{\partial y}=0, \quad \frac{\partial T_{G}}{\partial x}=0, \quad \frac{\partial T_{S}}{\partial x}=0 \quad \text { and } \quad \frac{\partial Y_{\eta}}{\partial x}=0
$$

\section{Outflow}

A zero normal gradient boundary condition was applied to $v$. Conversely, due to the conservation of mass principle, the axial outflow velocity was calculated such that $\left(\rho_{G} u\right)_{\text {out }}=\dot{m}_{\text {in }}$. Zero normal gradient was assumed for both temperature and gas species equations.

\section{Plain-porous medium interface}

According to [25], different types of boundary condition at the plain-porous medium interface (the transition between gas flow in the channel and gas flow within the porous wall) have negligible effects on the overall model results. Therefore, no boundary condition was applied in plain-porous interfaces for gas phase momentum and gas phase energy equations, i.e. the model would automatically set porosity to 1 and the BrinkmanForchheimer-extended Darcy term to zero (momentum equation only) when the 
calculations were performed in the free-stream. Similarly, for porous media, the Brinkman-Forchheimer-extended Darcy term was added and porosity was set according to which porous medium was in consideration. This technique has been used by several researchers ([26] and [27]). Zero normal gradients were assumed for the gas species calculations and as for solid temperature, the following boundary condition is used

$-\phi k_{G, p} \frac{\partial T_{G, p}}{\partial y}-\phi k_{S} \frac{\partial T_{S}}{\partial y}=h\left(T_{G}-T_{G-S}\right)$

where $T_{G-S}$ is the temperature at plain-porous media interface.

\section{EXPERIMENTAL DETERMINATION OF INTERSTITIAL HEAT TRANSFER COEFFICIENT}

In order to better predict the DPF temperature profile during the regeneration process, it is important to understand the heat transfer between the gas and solid phases inside the porous wall. A survey of the literature indicated that, to the authors' knowledge, no experiment that estimates the heat transfer coefficient inside a porous filter of a diesel particulate filter has so far been reported. Therefore, this section describes specific heating and cooling experiments on a DPF. It will be followed by an analysis of the data so that the interstitial heat transfer coefficient can be obtained.

The experiments were carried out using a hot flow rig that provides a controlled flow into an instrumented DPF. The set-up of the experiment is illustrated in Figure 2.

$<$ Figure 2>

A centrifugal air blower supplied air flow to the hot flow rig. The flow rate was controlled by a series of ball valves and measured using a pitot tube flowmeter. The air flow was heated up by an electric resistance heater, which was connected to a closedloop controller. The hot air then entered into the test rig and exited through the DPF outlet channels. 
The pressure reading at the upstream of the DPF was measured using a Digital Manometer with an accuracy of $\pm 10 \mathrm{~Pa}$. The temperature profiles of the DPF were measured by eight, $0.5 \mathrm{~mm}$ diameter $\mathrm{K}$ - type thermocouples with an accuracy of $\pm 0.5{ }^{\circ} \mathrm{C}$. Thermocouples measured the upstream and downstream gas temperature while the others measured the gas temperatures at three different locations along the filter length. At each location, the gas temperatures within the inlet and outlet channels of the DPF were measured as shown in Figure 3. The thermocouples were connected to an eight-channel temperature data logger. The data logger was calibrated by the manufacturer with a quoted accuracy of $\pm 0.1 \%$. The channel gas temperature history was recorded for the heating-up and cooling-down periods. This procedure was repeated for several different inlet temperatures and flow rates.

$<$ Figure 3 $>$

The specification of the DPF used in the experimental testing is summarised in Table 1:

$<$ Table 1>

Experimental runs were conducted to test the thermal response of a clean DPF during the heating and cooling processes for each test condition. Experiments were carried out under the following test conditions given in Table 2.

$<$ Table $2>$

The inlet gas temperature was increased to the desired level and held constant until steady-state was reached. The air heater was then switched off. The heating and cooling phases at various locations were recorded. Figure 4 shows the thermocouple response for an inlet temperature of $400{ }^{\circ} \mathrm{C}$. Similar results were obtained for other inlet temperatures and these can be found in [30].

$<$ Figure 4> 


\section{NUMERICAL SOLUTION PROCEDURE}

The aforementioned governing equations (1)-(15), were discretized by the finite volume method [28]. To avoid the non-physical 'checker-box' pattern of velocity field, a staggered grid was used [28]. Equations (1)-(15) were then solved by PISO scheme, proposed by Issa [29]. The details of the numerical procedure can be found in [30].

\section{RESULTS AND DISCUSSIONS}

\subsection{Determination of Interstitial Heat Transfer Coefficient}

By reassessing transient and steady-state experimental results by several investigators in [31], Wakao and Kaguei (1982) found that both the transient and steady-state data can be represented by a single correlation of the form $\mathrm{Nu}=f\left(\operatorname{Pr}^{m_{1}} \operatorname{Re}^{m_{2}}\right)$, where the indices $m_{1}$ and $m_{2}$ are empirically defined. Therefore, transient heat transfer calculations can be approximated by a steady-state analysis.

The heat transfer between the solid and gas phase of a porous DPF wall can be simplified as a 1-D conduction and convection model in the direction of gas flow through the wall. The gas temperature at the inlet side of the porous wall, $T_{\text {inlet }}$, at the outlet side of the porous wall, $T_{\text {outlet }}$ and the wall thickness $\Delta y_{f y}$ can be measured. By assuming a square channel with uniform heat flux both in the flow direction and around the periphery, i.e. $\mathrm{Nu}=3.091$ [32], and that the hydrodynamic length of the channel equals its width, the convective heat transfer coefficient in a square channel is approximately $87 \mathrm{~W} \cdot \mathrm{m}^{-2} \cdot \mathrm{K}^{-1}$. Assuming the characteristic length of the model equals the DPF wall thickness, the Biot number, $\mathrm{Bi}=0.024<0.1$. Hence, the heat transfer of a DPF can be analysed as a lumped-heat-capacity system [32].

The total power to the DPF during steady state can be written as

$$
Q_{i n}=\frac{\Delta T}{\sum R_{t b}}=\frac{T_{\text {inlet }}-T_{\text {outlet }}}{\sum R_{t b}}
$$


where

$$
\begin{aligned}
\sum \mathrm{R}_{t b} & =\frac{1}{b_{\text {inlet }} A_{f i l}}+\frac{1}{b_{\text {interstitia }} A_{\text {int }}}+\frac{\Delta y_{f w}}{k_{f v} \cdot A_{\text {int }}}+\frac{1}{b_{\text {outlet }} A_{f i l}} \\
& =\frac{2}{b_{\text {inlet }} A_{f i l}}+\frac{1}{b_{\text {interstitia }} A_{\text {int }}}+\frac{\Delta y_{f w}}{k_{f v} \cdot A_{\text {int }}}
\end{aligned}
$$

and $b_{\text {inlet }}, h_{\text {outlet }}$ are the convective heat transfer coefficients for the inlet and outlet channels, and $h_{\text {interstitial }}$ is the convective heat transfer coefficient for the porous medium, $A_{f i l}, A_{\text {int }}$ are the filtration area and the area inside porous wall, $\Delta y_{f y}$ is the thickness of DPF wall, and $k_{f w}$ is the effective DPF wall conductivity.

Here the conduction and convection heat transfer processes in the porous wall are in series and the convective heat transfer coefficients for both inlet and outlet channels are the same. The specific area of the porous wall is

$$
a_{f w}=4 \phi_{f i v} / d_{\text {pore }}
$$

and the internal surface area can be calculated from

$$
A_{\text {int }}=a \cdot A_{f i l} \cdot \Delta y_{f w}
$$

The inlet power into the control volume (DPF) concerned, can be calculated by

$$
Q_{\text {in }}=\dot{m}_{\text {inlet }} c_{p G}\left(T_{\text {inlet }}-T_{\text {outlet }}\right)
$$

Equating (19) and (23) and simplifying yields the interstitial heat transfer coefficient as

$$
b_{\text {interstitial }}=\frac{1}{\left[\frac{1}{\dot{m}_{\text {inlet }} c_{p G}}-\left(\frac{2}{b_{\text {inlet }} A_{f i l}}+\frac{\Delta y_{f w}}{k_{f w} \cdot A_{\text {int }}}\right)\right] A_{\text {int }}}
$$


Figure 6 shows the temperature differences between the DPF inlet and outlet channels for an inlet temperature of $400{ }^{\circ} \mathrm{C}$. Generally, it can be observed that temperature differences are directly proportional to the magnitude of the inlet temperature as well as the distance from the front face of the DPF.

$<$ Figure 6>

Linear regression of the Nusselt number, $\mathrm{Nu}$, as a function of $\left(\operatorname{Pr}^{m_{1}} \operatorname{Re}^{m_{2}}\right)$ allowed the calculation of the interstitial heat transfer coefficient for the experimental conditions of interest. The interstitial heat transfer coefficient of the filter wall was found to be $8.30 \mathrm{~W} \cdot \mathrm{m}^{-2} \cdot \mathrm{K}^{-1}$. Detail of the analysis can be found in [30]. The mass transfer coefficient can be calculated (assuming unity for the Lewis number) as

$$
k_{m}=\frac{h_{\text {intersitital }}}{\rho_{G} c_{p G}}
$$

The empirically derived value of $h_{\text {intersitial }}$ was used in the previous 1-D model [11]. It was found that $b_{\text {interstital }}$ is large enough that the heat transfer in the DPF is relatively insensitive to the interstitial heat transfer coefficient when compared to, for example, flow rate. This is discussed in more detail when considering thermal equilibrium discussions in sub-section 5.3.2. Figure 7 shows the comparison of 1-D model result (Equation 19) with the experimental data from [33].

$<$ Figure $7>$

\subsection{Model Comparisons}

Experimental data from [33] were used to validate the 2-D wall-flow DPF regeneration model. A grid density of 60 by 60 in both $\mathrm{x}$ - and y-directions was used in the simulation. For stability, a time-step of $2.5 \times 10^{-5} \mathrm{sec}$ was used in all simulation runs. The grid sizes were chosen such that the simulation results were grid independent. 
It should be emphasized that no arbitrarily-modified input parameters were used in the simulations. This is unusual in DPF regeneration modelling. All input parameters were based on experimentally-determined data. Therefore, it is assumed that the discrepancy between the model and the experimental measurements can be mainly attributed to the physics or chemistry not considered in the model. In addition, it should be noted that errors could occur in the experimental measurements and data. Therefore, full validation of the 2-D model result was not possible and the model was validated against available experimental data from [??].

A comparison between the 2-D model and the experimental results by [33] at different DPF locations is shown in Figure 8. The measurement locations $T_{1}$ to $T_{5}$ are shown in Figure 9. The input data used in this 2-D model is identical to that used in the previous 1-D model [11], except the PM porosity was set to 0.92 in agreement with [34]. Figure 8 shows that the 2-D model is able to predict the gas temperature in the DPF reasonably well. However, the model under-predicts the magnitude of the gas temperature. For locations $\mathrm{T}_{2}$ and $\mathrm{T}_{3}$ (see Figure 9), the temperature differences are approximately $100 \mathrm{~K}$ and $50 \mathrm{~K}$. Figure 8 also shows that the model predicts an outlet temperature which is close to that in the experimental data at $\mathrm{T}_{3}$. In addition, the $2-\mathrm{D}$ model predicts a slower initial heating rate compared with the experimental data for locations $T_{2}$ and $T_{3}$. The difference could be due to the heat liberated from the PM soluble organic fraction (SOF) vaporization during the initial heating up period, since the diesel PM used in [33] was not preheated prior to the DPF regeneration process. In addition, the model predicts a faster temperature reduction rate after the PM oxidation. To explain this, it is worth noting that in reality, during the regeneration process, the diesel PM is not uniformly oxidised in the DPF. Therefore, the slower temperature drop shown in the experimental data could be due to the heat addition from other channels. In spite of these discrepancies, the model nevertheless predicts the outlet gas temperature profiles well. For example, it shows the highest gas temperature at the downstream end of the DPF which is due to the heat accumulation in the DPF.

$<$ Figure 8>

$<$ Figure $9>$ 


\subsection{The Simulated DPF Regeneration Process}

\subsubsection{Flow Field Profile}

Figure 10 shows the exhaust flow profile inside the computational domain. Generally, the exhaust gas flows in a parabolic profile in the inlet and outlet channels whereas it is one-dimensional inside the porous diesel PM layer and DPF filter wall. In addition, it can be observed that upon entering the inlet channel, the exhaust gas flows in the direction towards the inlet symmetry line which was anticipated as the gas flow is constricted from the open exhaust flow upon entry to the channel inlets. The same observation was reported by Hou and Angelo [14]. This type of profile cannot be obtained by a 1-D model.

Figure 10 shows a velocity increase in the PM layer because the corresponding PM is fully oxidised at $t=30 \mathrm{sec}$. As the regeneration proceeds, recirculation starts to appear at the high temperature region e.g. $t=55$ to $60 \mathrm{sec}$ and flows toward the inlet channel end. The recirculation ceases toward the end of regeneration process when the diesel PM is fully oxidised. Another important finding that can be drawn from Figure 10 is that the flow field profile in the inlet channel is not the mirror image of that in outlet channels and vice-versa, which is often assumed in other existing models [35].

$<$ Figure 10>

\subsubsection{Gas and Solid Temperature Profiles}

Initially the DPF is heated up by the hot exhaust gas flow. The exhaust gas temperature increases from upstream to downstream ends. For the same axial location, the outlet channel is heated quicker compared to that in the inlet channel $(t=20-25 \mathrm{sec}$, Figure 11). At $t=30 \mathrm{sec}$, while the DPF is being heated up, the gas temperature at the upstream end is sufficiently high to cause an appreciable amount of PM to oxidise and results in a sudden increase of gas temperature at the upstream end. From Figure 11, it can be observed that the gas temperature increases appreciably as the high temperature zone flows from the DPF upstream to the downstream. The locally high gas temperature starts to cool down when the remaining diesel PM is fully consumed at $t=70 \mathrm{sec}$. 
$<$ Figure $11>$

The temperature in the PM layer and DPF wall follow the same trends as the gas phase as a consequence of the close coupling of the gas and solid phase temperatures through the relatively high interstitial heat transfer coefficient. Therefore, the solid temperature profiles are not plotted here. Instead, attention is paid in assessing the validity of TE assumption that is often used in many of the existing models such as [10, 14]. These authors argued that due to high specific area inside the DPF porous structure, TE could be confidently used in the solid temperature calculation. In the current study, the thermal equilibrium assumption was assessed by examining the gas and solid temperatures at six locations within the DPF wall. These locations are marked by white circles as shown in Figure 12. The gas and solid temperatures at these locations are shown in Figure 13. From Figure 13 (a), it can be observed that gas and solid phases have similar temperature magnitudes up to $1000 \mathrm{~K}$. The temperature differences become larger when the gas and solid temperatures reach beyond $1000 \mathrm{~K}$ or during the cooling stage. During cooling, it can be noticed that the gas temperatures fall quicker than those of the solid. At these locations, a maximum temperature difference of $37 \mathrm{~K}$ is observed. The coupling between the solid and gas phase temperature is a consequence of the interstitial heat transfer coefficient, and has a direct impact on the validity of thermal equilibrium modelling assumptions employed by some authors.

The gas and solid temperature differences at location $(j 2+\mathrm{kmax})$ are more pronounced compared to those at location (j2+1) (see Figure 13 (b)). A maximum temperature difference of $56 \mathrm{~K}$ can be observed at location ( $\mathrm{j} 2+\mathrm{kmax})$. Hence, this study shows that TE does not hold for the DPF regeneration process.

$<$ Figure 13>

\subsubsection{Gas Species Profiles}

\section{(1) Oxygen}

Figure 14 shows the oxygen mass fraction distributions in the DPF during the regeneration process. At the beginning of the regeneration process, uniform oxygen was assumed in the computational domain. As the gas temperature increases, this initiates PM oxidation which consumes oxygen. It can be observed that the region of lower oxygen 
content propagated from the DPF upstream to the downstream side. When the PM is fully consumed, the oxygen consumption ceases and therefore the oxygen content increases due to the oxygen supply from the inlet channel. The oxygen consumption and recovery processes continued until all the PM was fully oxidised at the end of regeneration process.

$<$ Figure 14>

\section{(2) Carbon Dioxide}

Since $\mathrm{CO}_{2}$ is the main overall product of PM oxidation with $\mathrm{O}_{2}$, it follows the opposite trend as that of $\mathrm{O}_{2}$. $\mathrm{CO}_{2}$ increased at the outlet channel as a result of increasing PM oxidation as well as conversion of $\mathrm{CO}$ to $\mathrm{CO}_{2}$. The development of local hot spots causes the local $\mathrm{CO}_{2}$ mass fraction to increase as high as 0.4. At the end of regeneration process, uniform $\mathrm{CO}_{2}$ distribution returns due to the inlet supply of $\mathrm{CO}_{2}$.

\section{(3) $\underline{\text { Carbon Monoxide }}$}

From Figure 16, it can be observed that the main CO generation path is via the PM oxidation since higher CO concentration is in the PM layer. Since the PM temperature is sufficiently high, it produces higher $\mathrm{CO}$ concentration in the form of a continuous stripe (e.g. $t=30$ to $40 \mathrm{sec}$ ). Several locally higher $\mathrm{CO}$ concentration spots rather than a continuous stripe are developed later (e.g. $t=50$ to $60 \mathrm{sec}$ ). Apart from direct conversion from PM oxidation, these could be due to the gas phase reactions which involve the CO production, such as R2 and R4. After all the PM is fully consumed, the uniform CO distribution in the DPF is recovered (i.e. tends to diesel exhaust levels).

$<$ Figure 16>

\section{(4) Water Vapour}

In the multi-step chemical reaction scheme, water vapour is only generated by the watergas shift reaction under high temperature environment. At $t=30 \mathrm{sec}$ (see Figure 17), the gas temperature becomes sufficiently high to initiate gas phase reaction and the $\mathrm{H}_{2} \mathrm{O}$ content increases at the DPF upstream. There is an uneven $\mathrm{H}_{2} \mathrm{O}$ distribution with a decrease of local $\mathrm{H}_{2} \mathrm{O}$ concentration at the end of DPF inlet channel $(t=40 \mathrm{sec})$. The 
high concentration of $\mathrm{H}_{2} \mathrm{O}$ follows the combustion wave (high gas temperature region) and causes an increase of $\mathrm{H}_{2} \mathrm{O}$ concentration at the DPF downstream $(t=50 \mathrm{sec})$. As the gas temperature decreases the $\mathrm{H}_{2} \mathrm{O}$ content decreases to the initial state $(t=60 \mathrm{sec})$. $<$ Figure $17>$

(5) Hydrogen

Similar to water vapour, $\mathrm{H}_{2}$ concentration variations are due to the water-gas shift (WGS) reaction. Due to its small molecular weight of $2 \mathrm{~g}^{\mathrm{mol}}{ }^{-1}$, its presence during DPF regeneration process is virtually negligible. $\mathrm{H}_{2}$ is only produced in high temperature conditions where local hot regions develop. In addition, it is only produced sporadically and is not present for a long time i.e. it is quickly converted to $\mathrm{H}_{2} \mathrm{O}$.

$<$ Figure 18>

\subsubsection{PM Oxidation Profile}

Figure 19 shows the amount of PM as a function of time and filter axial length. From Figure 19(a), a noticeable PM oxidation rate occurs at about $t=12 \mathrm{sec}$. After that, the oxidation rate increases with the highest oxidation rate occurring at $t=30 \mathrm{sec}$. This rapid oxidation rate decreases at around $t=45 \mathrm{sec}$. The PM is fully oxidised at $t=69 \mathrm{sec}$.

From Figure 19(b) it shows that more PM is initially consumed at the upstream end of the DPF, which is nearer to the high temperature inlet. Therefore, the PM is consumed in a wave-like form rather than as a uniformly shrinking layer, until all the PM is fully oxidised.

$<$ Figure 19> 


\section{CONCLUSIONS}

A new 2-D DPF regeneration model has been presented, including an experimental study to estimate the interstitial heat transfer coefficient inside DPF porous wall. Several conclusions can be drawn from these studies and they are summarised as follows:

1. Experimental work was carried out to estimate the interstitial heat transfer coefficient in the DPF filter wall and was found to be $8.30 \mathrm{~W} \cdot \mathrm{m}^{-2} \cdot \mathrm{K}^{-1}$.

2. A new 2-D finite volume method based DPF regeneration model, which includes a multi-step reaction scheme, has been developed. The model was compared quantitatively and qualitatively with experimental data as well as modelling findings reported in the literature.

3. The 2-D model showed that the velocity magnitude is largest where exhaust gas immediately enters into the inlet channel and also when the exhaust gas leaves through the outlet channel.

4. The model predicted that recirculation occurs in the DPF during the regeneration process. The initiation of the recirculation is associated with the high gas temperature region.

5. The simulated flow field profile in the inlet channel is not the mirror image of that in the outlet channel and vice-versa as has been assumed in some previous models.

6. The model predicted that that the high temperature zone moves from the upstream to the downstream of the DPF. When the exhaust gas temperature is sufficiently high and uniform in the filter, fast reactions occur and heat energy from the chemical reactions is released and quickly propagates to the downstream end of the filter.

7. The thermal equilibrium assumption used by many previous modellers has been examined and found to be violated in the case of a high PM oxidation rate. Thus, the models presented here, which implement NTE, are considered to be more realistic because thermal equilibrium was not assumed. 
8. Various species distribution profiles have been studied during the regeneration process. Generally the species distributions depend on the PM oxidation rates and the species availability within local gas.

9. The 2-D model predicted that PM layer was consumed in a wave-like form during the regeneration process.

\section{ACKNOWLEDGEMENTS}

The authors gratefully acknowledge Loughborough University and DTI/DfT Foresight Vehicle 'LOCOFILT' Programme for supporting this research. We also thank Dr. Cornelius Opris of Caterpillar Inc. for his advice. Perkins Engines and the Royal Academy of Engineering are also gratefully acknowledged for supporting the corresponding author. 


\section{LIST OF TABLES AND FIGURES}

Table 1 DPF specifications

Table 2 Experimental conditions.

Figure 1 Computational domain, consists of inlet, outlet channel as well as the porous filter wall.

Figure 2 Schematic of experimental set - up.

Figure 3 Schematic of thermocouples arrangement in a 5.66 inch diameter DPF.

Figure 4 DPF temperature responses for inlet temperature $400{ }^{\circ} \mathrm{C}$

Figure 5 Schematic of DPF model.

Figure 6 Temperature differences for inlet temperatures of $400{ }^{\circ} \mathrm{C}$

Figure 7 Comparison of model and experimental data.

Figure 8 Comparison between experimental data and model result with different axial locations

Figure 9 Thermocouple locations in wall flow DPF used in [33]

Figure 10 Velocity field profiles during the regeneration process

Figure 11 Gas temperature history during regeneration process

Figure 12 Monitoring locations for gas and solid temperatures

Figure 13 Gas and solid temperature difference at various points at layers (a) $\mathrm{j} 2+1$

(b) $\mathrm{j} 2+$ kmax

Figure $14 \mathrm{O}_{2}$ mass fraction distributions during regeneration process

Figure $15 \mathrm{CO}_{2}$ mass fraction distributions during regeneration process

Figure $16 \mathrm{CO}$ mass fraction distributions during regeneration process

Figure $17 \mathrm{H}_{2} \mathrm{O}$ mass fraction distributions during regeneration process

Figure $18 \mathrm{H}_{2}$ mass fraction distributions during regeneration process

Figure 19 Remaining PM profiles: (a) as the function of time, and (b) along the filter length 


\section{Nomenclature}

$a$

$A_{\text {fil }}$

$A_{\text {int }}$

$c_{p}$

$C_{E}$

$d$

D

$b$

$h_{\text {interstitial }}$

$b_{\text {inlet }}$

$h_{\text {outlet }}$

$H$

$k_{f w}$

$k_{\mathrm{G}}$

$k_{\mathrm{m}}$

$k_{\mathrm{s}}$

K

$L$

$m$

$M W$

$p$ specific area, $\left(\mathrm{m}^{-1}\right)$

total filtration area, $\left(\mathrm{m}^{2}\right)$

total area inside porous wall, $\left(\mathrm{m}^{2}\right)$

specific heat capacity at constant pressure, $\left(\mathrm{J} \cdot \mathrm{kg}^{-1} \cdot \mathrm{K}^{-1}\right)$

coefficient in modified Darcy's model, (-)

diameter, $(\mathrm{m})$

diffusion coefficient, $\left(\mathrm{m}^{2} \cdot \mathrm{s}^{-1}\right)$

heat transfer coefficient, $\left(\mathrm{W} \cdot \mathrm{m}^{-2} \cdot \mathrm{K}^{-1}\right)$

interstitial heat transfer coefficient, $\left(\mathrm{W} \cdot \mathrm{m}^{-2} \cdot \mathrm{K}^{-1}\right)$

heat transfer coefficient inside inlet channel, $\left(\mathrm{W} \cdot \mathrm{m}^{-2} \cdot \mathrm{K}^{-1}\right)$

heat transfer coefficient inside outlet channel, $\left(\mathrm{W} \cdot \mathrm{m}^{-2} \cdot \mathrm{K}^{-1}\right)$

enthalpy of reaction, $\left(\mathrm{J} \cdot \mathrm{mol}^{-1}\right)$

thermal conductivity of a DPF, $\left(\mathrm{W} \cdot \mathrm{m}^{-1} \cdot \mathrm{K}^{-1}\right)$

gas phase conductivity, $\left(\mathrm{W} \cdot \mathrm{m}^{-1} \cdot \mathrm{K}^{-1}\right)$

mass transfer coefficient, $\left(\mathrm{m} \cdot \mathrm{s}^{-1}\right)$

solid phase conductivity, (W. $\left.\mathrm{m}^{-1} \cdot \mathrm{K}^{-1}\right)$

permeability of porous media, $\left(\mathrm{m}^{2}\right)$

characteristic length, $(\mathrm{m})$

mass, $(\mathrm{kg})$

molecular weight, $\left(\mathrm{kg} \cdot \mathrm{mol}^{-1}\right)$

pressure, $(\mathrm{Pa})$ 


$\begin{array}{ll}Q & \text { power, }(\mathrm{W}) \\ \dot{\mathrm{R}}^{\prime \prime \prime} & \text { species production } / \text { consumption rate, }\left(\mathrm{mol} \cdot \mathrm{m}^{-3} \cdot \mathrm{s}^{-1}\right) \\ \mathrm{R}_{G} & \text { gas constant, }\left(\mathrm{J} \cdot \mathrm{mol}^{-1} \cdot \mathrm{K}^{-1}\right) \\ \mathrm{R}_{t b} & \text { thermal resistance, }\left(\mathrm{m}^{2} \cdot \mathrm{K} \cdot \mathrm{W}^{-1}\right) \\ \mathrm{R}_{u} & \text { universal gas constant, } 8.3142\left(\mathrm{~J} \cdot \mathrm{mol}^{-1} \cdot \mathrm{K}^{-1}\right) \\ t & \text { time, }(\mathrm{s}) \\ T & \text { temperature, }(\mathrm{K}) \\ u & \text { axial gas velocity, }\left(m \cdot \mathrm{s}^{-1}\right) \\ v & \text { radial gas velocity, }\left(m \cdot \mathrm{s}^{-1}\right) \\ x & \text { axial direction, }(-) \\ y, z & \text { radial direction used in } 2-\mathrm{D} \text { and 1-D models respectively, (-) } \\ Y & \text { mass fraction, }(-)\end{array}$

\section{Acronyms}

1-D

2-D

3-D

DPF

NTE

PDE

PISO

PM

SOF

$\mathrm{T} / \mathrm{C}$

TE

WGS one-dimensional

two-dimensional

three-dimensional

diesel particulate filter

non-thermal equilibrium

partial differential equation

pressure implicit with splitting of operators

particulates matter

soluble organic fraction

thermocouple

thermal equilibrium

water-gas shift 


\section{Non-dimensional numbers}

$\begin{array}{ll}\mathrm{Bi} & \text { Biot number, }=h . L / k_{s} \\ \mathrm{Le} & \text { Lewis number, }=h /\left(c_{p G} \cdot g_{p}\right) \\ \mathrm{Nu} & \text { Nusselt number, }=h . L / k_{G} \\ \mathrm{Pr} & \text { Prandtl number, }=c_{p G} \cdot \mu_{G} / k_{G} \\ \mathrm{Re} & \text { Reynold number, }=\rho_{G} u L / \mu_{G}\end{array}$

\section{Greek letters}

$\begin{array}{ll}\alpha & \alpha^{t h} \text { reaction }(-) \\ \beta_{p} & \text { bulk density of particulate, }\left(\mathrm{kg} \cdot \mathrm{m}^{-3}\right) \\ \chi_{\eta} & \text { mole fraction of species } \eta \\ \Delta & \text { difference of a quantity, }(-) \\ \rho & \text { density, }\left(\mathrm{kg} \cdot \mathrm{m}^{-3}\right) \\ \mu & \text { viscosity, }\left(\mathrm{kg} \cdot \mathrm{m}^{-1} \cdot \mathrm{s}^{-1}\right) \\ \tau & \text { tortuosity of porous media, }(-) \\ \phi & \text { porosity of porous media, }(-) \\ \eta, \gamma & \text { general gas constituent, }(-) \\ v_{\eta \alpha} & \text { stoichiometric coefficient of a species } \eta \text { in a reaction } \alpha,(-) \\ v^{\prime}, v^{\prime \prime} & \text { gas reactant stoichiometric coefficient, }(-)\end{array}$

\section{Matrix-Vector quantities}

$\mathbf{U}$

velocity vector, $\left(m \cdot s^{-1}\right)$

\section{Subscripts}

D

Darcian

eff

effective 


$\begin{array}{ll}f w & \text { filter wall } \\ G & \text { gas phase } \\ \text { in } & \text { input } \\ p & \text { particulate } \\ \text { pore } & \text { pore in ceramic substrate } \\ S & \text { solid phase } \\ \text { symmetry } & \text { symmetrical line of the inlet or outlet channels } \\ \eta m & \text { of species } \eta \text { in the gas mixture }\end{array}$

\section{Superscripts}

$\begin{array}{ll}m_{1}, m_{2} & \text { indices used in curve-fitting }(-) \\ / / & \text { per unit area }\left(\mathrm{m}^{-2}\right) \\ / / & \text { per unit volume }\left(\mathrm{m}^{-3}\right) \\ \text {. } & \text { derivative with respect to time }\end{array}$




\section{References}

1. Wallace, W., Liu, L., Keane, M., Cui, M., Ensell, M., Miller, W., Kashon, K., and Ong, T. In Vitro Genotoxicity of Gasoline and Diesel Engine Vebicle Exhaust Particulate and Semi-Volatile Organic Compound Materials. in $8^{\text {th }}$ Diesel Engine Emission Reduction Conference. 2002. San Diego, CA.

2. Hall, D.E., King, D.J., Morgan, T.D.B., Baverstock, S.J., Heinze, P., and Simpson, B.J., A Review of Recent Literature Investigating the Measurement of Automotive Particulate; the Relationship with Environmental Aerosol, Air Quality and Health Effects. SAE 982602, 1998.

3. Khair, M.K., A Review of Diesel Particulate Filter Technologies. SAE Paper 2003-01-2303, 2003.

4. Bissett, E.J., Mathematical Model of the Thermal Regeneration of a Wall-Flow Monolith Diesel Particulate Filter. Chemical Engineering Science, 1984. 39(7/8): p. 1233-1244.

5. Garner, C.P. and Dent, J.C., A Thermal Regeneration Model for Monolithic and Fibrous Diesel Particulate Traps. SAE Transactions, Journal of Passenger Cars, 1988. 97(4): p. 9-24.

6. Aoki, H., Asano, A., Kurazono, K., Kobashi, K., and Sami, H., Numerical Simulation Model for the Regeneration Process of a Wall-Flow Monolith Diesel Particulate Filter. SAE Paper 930364, 1993.

7. Opris, C.N. and Johnson, J.H., A 2-D Computational Model Describing the Heat Transfer, Reaction Kinetics and Regeneration Characteristics of a Ceramic Diesel Particulate Trap. SAE Paper 980546, 1998.

8. Konstandopolous, A.G., Kostoglou, M., Housiada, P., Vlachos, N., and Zarvalis, D., Multichannel Simulation of Soot Oxidation in Diesel Particulate Filters. SAE Paper 2003-01-0839, 2003.

9. Koltsakis, G.C., Haralampous, O.A., Margaritis, N.K., Samaras, Z.C., Vogt, C.-D., Ohara, E., Watanabe, Y., and Mizutani, T., 3-Dimensional Modeling of the Regeneration in SiC Particulate Filters. SAE Paper 2005-01-0953, 2005.

10. Zheng, H. and Keith, J.M., Ignition Analysis of Wall-Flow Monolith Diesel Particulate Filters. Catalysis Today, 2004. 98: p. 403-412.

11. Law, M.C., Clarke, A., and Garner, C.P., A Diesel Particulate Filter Regeneration Model with a Multi-Step Chemical Reaction Scheme. Proceedings of the IMechE Part D: Journal of Automobile Engineering, 2005. 219(D2): p. 215-226. 
12. Pontikakis, G., Stamatelos, A., Bakasis, K., and Aravas, N., 3-D Catalytic Regeneration and Stress Modeling of Diesel Particulate Filters by Abaqus Fem Software. SAE Paper 2002-01-1017, 2002.

13. Bissett, E.J. and Shadman, F., Thermal Regeneration of Diesel Particulate Monolithic Filters. AICbE Journal, 1985. 31(5): p. 753-758.

14. Hou, J.Z. and Angelo, T. A New Cfd Model for Understanding and Managing Diesel Particulate Filter Regeneration. in $10^{\text {th }}$ Diesel Engine Emissions Reduction Conference. 2004. Coronado, California.

15. Kaviany, M., Principles of Heat Transfer in Porous Media. Second ed, ed. F.F. Ling. 1999, New York: Springer-Verlag New York, Inc.

16. Konstandopolous, A.G., Kostoglou, M., Skaperdas, E., Papaioannou, E., Zarvalis, D., and Kladopoulou, E., Fundamental Studies of Diesel Particulate Filters: Transient Loading, Regeneration and Aging. SAE 2000-01-1016, 2000: p. $1-23$.

17. Konstandopolous, A.G., Skaperdas, E., and Masoudi, M., Inertial Contributions to the Pressure Drop of Diesel Particulate Filters. SAE Paper 200101-0909, 2001.

18. Alazmi, B. and Vafai, K., Constant Wall Heat Boundary Conditions in Porous Media under Local Thermal Non-Equilibrium Conditions. International Journal of Heat and Mass Transfer, 2002. 45: p. 3071-3087.

19. Law, M.C., Clarke, A., and Garner, C.P., The Effects of Soot Properties on the Regeneration Behaviour of Wall-Flow Diesel Particulate Filters. Proceedings of the IMechE Part D: Journal of Automobile Engineering, 2004. 218(D12): p. 1513-1524.

20. Turns, S.R., An Introduction to Combustion. Second Edition ed. 2000, USA: McGraw-Hill International Editions. 676.

21. Haralampous, O.A., Koltsakis, G.C., Samaras, Z.C., Vogt, C.-D., Ohara, E., Watanabe, Y., and Mizutani, T., Reaction and Diffusion Phenomena in Catalyzed Diesel Particulate Filters. SAE Paper 2004-01-0696, 2004.

22. Hayes, R.E. and Kolaczkowski, S.T., Introduction to Catalytic Combustion. 1997, Amsterdam: Gordon and Breach Science Publishers.

23. Satterfield, C.N., Mass Transfer in Heterogeneous Catalysis. 1970, Clinton, Massachusetts: The Colonial Press, Inc.

24. Ferziger, J.H. and Peric, M., Computational Methods for Fluid Dynamics. Third ed. 2002, Berlin: Springer-Verlag.

25. Alazmi, B. and Vafai, K., Analysis of Fluid Flow and Heat Transfer Interfacial Conditions between a Porous Medium and a Fluid Layer. International Journal of Heat and Fluid Flow, 2001. 44: p. 1735 - 1749. 
26. Zhang, H.Y. and Huang, X.Y., Volumetric Heat Transfer Coefficients in Solid-Fluid Porous Media: Closure Problem, Thermal Analysis and Model Improvement with Fluid Flow. International Communications in Heat and Mass Transfer, 2000. 43: p. 3417-3432.

27. Goyeau, B., Lhuillier, D., Gobin, D., and Velarde, M.G., Momentum Transfer at a Fluid-Porous Interface. International Journal of Heat and Mass Transfer, 2003. 46: p. 4071 - 4081.

28. Versteeg, H.K. and Malalasekera, W., An Introduction Computational Fluid Dynamics: The Finite Volume Method. 1995, Essex: Person Education Limited.

29. Issa, R.I., Solution of the Implicitly Discretised Fluid Flow Equations by Operator-Splitting. Journal of Computational Physics, 1985. 62: p. 40-65.

30. Law, M.C., Modelling $W$ all-Flow Diesel Particulate Filter Regeneration Processes, $\mathrm{PhD}$ Thesis, 2006, Loughborough University

31. Wakao, N. and Kaguei, S., Heat and Mass Transfer in Packed Beds. Topics in Chemical Engineering, ed. R. Hughes. 1982, London: Gordon and Breach, Science Publishers Ltd.

32. Holman, J.P., Heat Transfer. $6^{\text {th }}$ ed. 1986, Singapore: McGraw Hill.

33. Higuchi, N., Mochida, S., and Kojima, M., Optimized Regeneration Conditions of Ceramic Honeycomb Diesel Particulate Filters. SAE Paper 830078, 1983.

34. Konstandopoulos, A.G., Skaperdas, E., and Mansoudi, M., Microstructural Properties of Soot Deposits in Diesel Particulate Traps. SAE Paper 2002-01-1015, 2002.

35. Guo, Z. and Zhang, Z., Multi-Dimensional Modelling and Simulation of WallFlow Diesel Particulate Filter During Loading and Regeneration. SAE Paper 2006-01-0265, 2006. 